\title{
MECHANICAL SYSTEM MODELLING OF ROBOT DYNAMICS USING A MASS/PULLEY MODEL
}

\author{
L. J. Stocco and M. J. Yedlin \\ The Department of Electrical and Computer Engineering, The University of British Columbia \\ 2332 Main Mall, Vancouver, BC, Canada, V6T $1 Z 4$ \\ leos@ece.ubc.ca,matty@ece.ubc.ca
}

\begin{abstract}
Keywords: Mass matrix, inertia matrix, MP model, pulley, differential transmission, mechanical system representation, robot dynamics, impedance, equivalent electric circuit.

Abstract: The well-known electro-mechanical analogy that equates current, voltage, resistance, inductance and capacitance to force, velocity, damping, spring constant and mass has a shortcoming in that mass can only be used to simulate a capacitor which has one terminal connected to ground. A new model that was previously proposed by the authors that combines a mass with a pulley (MP) is shown to simulate a capacitor in the general case. This new MP model is used to model the off-diagonal elements of a mass matrix so that devices whose effective mass is coupled between more than one actuator can be represented by a mechanical system diagram that is topographically parallel to its equivalent electric circuit model. Specific examples of this technique are presented to demonstrate how a mechanical model can be derived for both a serial and a parallel robot with both two and three degrees of freedom. The technique, however, is extensible to any number of degrees of freedom.
\end{abstract}

\section{INTRODUCTION}

The concept of impedance and its generalization reactance, has been used to define equivalent circuits of mechanical and electro-mechanical systems since the development of the Maxwell model of solids. The idea that driving point impedances could be decomposed into terms that parallel electrical elements was initiated by (Foster, 1924) who showed that the frequency response of any system is determined by the poles and zeros of its transfer function. The conditions for network synthesis are described by (Brune, 1931) and later applied by (Paynter 1961) who introduced bond graphs to distinguish and represent effort and flow variables in a graphical setting. Examples of electro-mechanical system simulations are numerous and include magnetic circuits (Hamill, 1993), mechatronics and electromechanical transducers (Tilmans, 1996), (van Amerongen \& Breedveld, 2003), (Sass et al., 2004).

Mechanical block diagrams are routinely used to model robot dynamics although some (Eppinger \& Seering, 1992) limit them to a single axis while others (Yamakita et al., 1992) rely entirely on equivalent electric circuits to avoid the inherent difficulties of creating mechanical models of multiaxis devices, transmission systems or other systems with coupled dynamics.

Section 2 of this paper describes the conventional electro-mechanical analogy and points out a limitation of the mass model. It goes on to describe a new mass/pulley (MP) model which overcomes the inherent deficiency in the conventional mass model. In Section 3, it is shown how the new MP model can be used to model the dynamics of devices which have coupled effective masses. Examples are provided which include both 2-DOF and 3-DOF serial and parallel manipulators. Lastly, concluding remarks are made in Section 4.

\section{ELECTRO-MECHANICAL ANALOGIES}

The ability to define an electro-mechanical equivalent circuit stems from the parallelism in the differential equations that describe electrical and mechanical systems, each of which involve an across variable, a through variable and an impedance or admittance variable. In electrical circuits, voltage $E(s)$ is the across variable and current $I(s)$ is the through variable. In mechanical systems, velocity $V(s)$ is the across variable and force $F(s)$ is the through variable (i.e. flow variable 
(Fairlie-Clarke, 1999)). This results in a correspondence between resistance $R$ and damping $B$, inductance $L$ and spring constant $K$, and capacitance $C$ and mass $M$ shown in (1-3). An alternate approach treats force as the across variable and velocity as the through variable but that approach is not used here. By (1-3), the electromechanical equivalents shown in Figure 1 can be substituted for one another to model a mechanical system as an electrical circuit and vise versa.

$$
\begin{array}{cr}
E(s)=I(s) R=I(s) \frac{1}{G} & V(s)=F(s) \frac{1}{B} \\
E(s)=I(s) s L & V(s)=F(s) \frac{s}{K} \\
E(s)=I(s) \frac{1}{s C} & V(s)=F(s) \frac{1}{s M}
\end{array}
$$

\begin{tabular}{|lll}
\hline & $F=I$ \\
\hline Roltage Source: $E(s)$ & Velocity Source: $V(s)$ \\
\hline
\end{tabular}

Figure 1: Admittance of electro-mechanical equivalents.

\subsection{Classical Mass Model Limitation}

Each of the components in Figure 1 has two terminals except for the mass which has only one. This is due to the fact that the dynamic equation of a mass (3) does not accommodate an arbitrary reference. Acceleration is always taken with respect to the global reference, or ground. Consider the two systems in Figure 2 which are well known to be analogous.

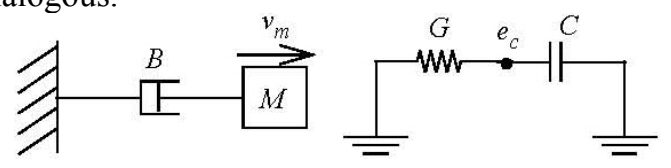

Figure 2: LC circuit and mechanical equivalent.

In Figure 2, the voltage across the capacitor $e_{c}$ corresponds to the velocity of the mass $v$. Both of these are relative measurements that only correspond to one another because both are taken with respect to ground. Consider, on the other hand, the circuit in Figure 3 which contains a capacitor with one terminal open circuited.
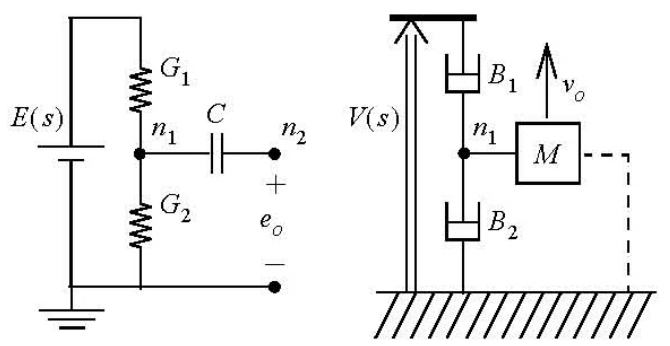

Figure 3: RC circuit and mechanical equivalent.

In Figure 3, the capacitor carries no current and therefore, has no effect on the output voltage $e_{o}$. In other words, the voltages at $n_{1}$ and $n_{2}$ are equal so the capacitor behaves like a short circuit. In the mechanical "equivalent", it is not possible to connect a non-zero mass $M$ to node $n_{1}$ without affecting the output velocity $v_{o}$. This is due to the implicit ground reference of the mass (shown by a dotted line) which prevents it from ever behaving like a mechanical short circuit. Note that this same limitation does not apply to the spring or damper since they both act as a mechanical short circuit (infinitely stiff connection) if one terminal is left unconnected, just like their electrical counterparts, the inductor and resistor.

\subsection{The Mass/Pulley (MP) Model}

Because of the above limitation, there are mechanical systems which can not be modelled using a mechanical system diagram. Elaborate transmission systems such as robotic manipulators may contain mass elements that are only present when relative motion occurs between individual motion stages. Currently, systems such as these can only be modelled using electric circuits since capacitors can be used to model this type of behaviour but masses cannot.

It would be useful to have a mechanical model which simulates the behaviour of a capacitor without an implicit ground connection so that any mechanism (or electric circuit) could be modelled by a mechanical system diagram. This new model should have two symmetric terminals (i.e. flipping the device over should not affect its response), obey Ohm's Law, and be able to accommodate non-zero velocities at both terminals simultaneously. A model proposed by the authors (Stocco \& Yedlin, 2006) combines a mass with the pulley-based differential 
transmission shown in Figure 4. The pulley system obeys the differential position / velocity relationship shown in $(4,5)$.

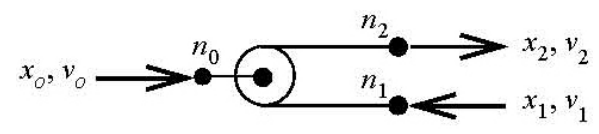

Figure 4: Pulley based differential transmission.

$$
\begin{gathered}
\Delta x_{0}=\frac{1}{2}\left(\Delta x_{2}-\Delta x_{1}\right) \\
v_{0}=\frac{1}{2}\left(v_{2}-v_{1}\right)
\end{gathered}
$$

Note from (5) that although the pulley provides the desired differential velocity input, it also introduces an undesired 2:1 reduction ratio. However, setting $v_{1}$ to 0 (i.e. connecting $n_{1}$ to ground) results in (6). Therefore, a similar pulley system with one input tied to ground could be used to scale up velocity by an equivalent ratio.

$$
v_{2}=2 v_{0}
$$

The double pulley system shown in Figure 5 is a differential transmission with a unity gear ratio. The primary pulley provides the differential input while the secondary pulley cancels the reduction ratio to achieve unity gain. A mass connected to the secondary pulley is accelerated by a rate equal to the difference between the acceleration of the two inputs, $n_{1}$ and $n_{2}$. This system simulates the behaviour of a capacitor that may or may not be connected to ground (Figure 5). Voltage $E_{1}$ corresponds to velocity $V_{l}$, voltage $E_{2}$ corresponds to velocity $V_{2}$, current $I$ corresponds to tension $F$ and capacitance $C$ corresponds to mass $M$ as shown by $(7,8)$. Note that the free-body diagram of the centre pulley shows that the tension $F$ in the primary cable is equal to the tension $F$ in the secondary cable. The system must be balanced because any net force on the massless centre pulley would result in infinite acceleration of the pulley and therefore, the mass as well.

$$
\begin{aligned}
& E_{2}(s)-E_{1}(s)=I(s) \frac{1}{s C} \\
& V_{2}(s)-V_{1}(s)=F(s) \frac{1}{s M}
\end{aligned}
$$

The MP model uses ideal cables with zero mass and infinite length and stiffness. The ideal cables travel through the system of massless, frictionless pulleys without any loss of energy. The
MP model operates in zero gravity so the mass is only accelerated as a result of cable tension and/or compression. Unlike practical cables, the ideal cables never become slack. When an attractive force is applied between $n_{1}$ and $n_{2}, F<0$ and the mass is accelerated downward. A block diagram of the MP model is presented in Figure 6 where $P$ has the same value as $M$ in Figure 5. Note that, unlike a pure mass, the MP model has two terminals, $n_{1}$ and $n_{2}$ which correspond to the two ends of the primary cable.

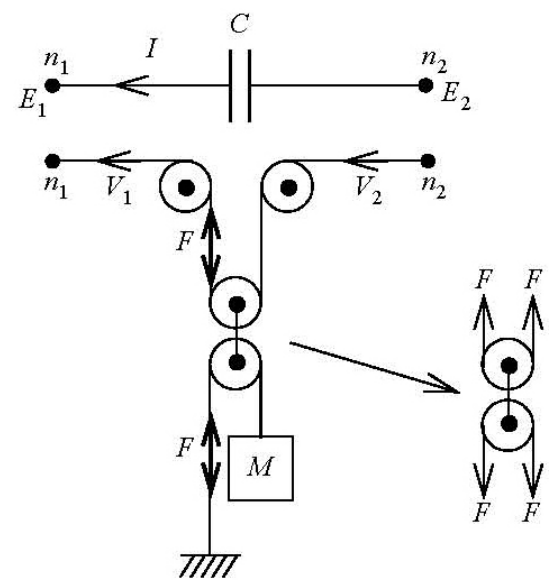

Figure 5: Mass / pulley equivalent of a capacitor.

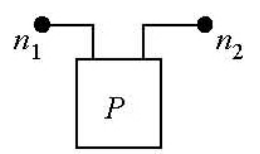

Figure 6: Block diagram of MP model.

Consider Figure 7 which is the mechanical system from Figure 3 with the mass replaced by an MP model. With terminal $n$, left unconnected, the primary cable of the MP model travels freely through the primary pulley without accelerating the mass or consuming energy. The MP model behaves like a mechanical short circuit, just like the capacitor in Figure 3. Also note the topological similarity between the electrical circuit in Figure 3 and its true mechanical equivalent in Figure 7. This is a direct result of the topological consistency between the capacitor and the MP model, both of which have two symmetric terminals. As pointed out in (Stocco \& Yedlin, 2006), this consistency allows one to analyze mechanical systems using electric circuit analysis techniques once all masses have been replaced by MP models. 


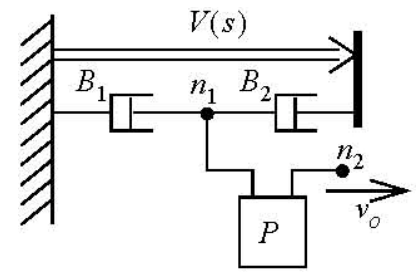

Figure 7: Mechanical equivalent using MP model.

\section{ROBOT MASS MATRIX}

Consider the simplified dynamics of a 2-DOF robot (9) where $M$ is the mass matrix, $B$ is the damping matrix, $F$ is a vector of joint forces/torques (10), $R$ is a vector of joint rates $r_{1}$ and $r_{2}(10)$, and $s$ is the Laplace operator. Spring constants, gravitational and coriolis effects are assumed to be negligible for the purpose of this example. If the damping in the system is dominated by the actuator damping coefficients, $B$ is a diagonal matrix (10). $M$, on the other hand, represents the effective mass perceived by each joint and is not diagonal or otherwise easily simplified in general.

$$
\begin{gathered}
F=B R+M s R \\
{\left[\begin{array}{l}
f_{1} \\
f_{2}
\end{array}\right]=\left[\begin{array}{ll}
b_{1} & 0 \\
0 & b_{2}
\end{array}\right]\left[\begin{array}{l}
r_{1} \\
r_{2}
\end{array}\right]+M s\left[\begin{array}{l}
r_{1} \\
r_{2}
\end{array}\right]}
\end{gathered}
$$

For simple kinematic arrangements such as the redundant actuators shown in Figure 8 which only have a single axis of motion, $M$ is shown in (11). The system responses are modeled by the mechanical system diagram shown in Figure 9 and the dynamic equation shown in (10). Using the electromechanical transformation described in Section 2, this system can also be represented by the electrical circuit analogy shown in Figure 9.

$$
M=\left[\begin{array}{ll}
m_{1} & m_{2} \\
m_{2} & m_{2}
\end{array}\right]
$$

Performing nodal analysis on the circuit in Figure 9 results in (12) by inspection. Note however, that (12) contains the term $i_{1}-i_{2}$ as well as $v_{2}$ which corresponds to the end-point velocity in the mechanical system or, in other words, the sum of the joint rates $r_{1}+r_{2}$. To obtain a correspondence between electrical and mechanical component values, the dynamic equation (10) is rearranged in (13) where the associated damping $B^{\prime}$ and mass $M^{\prime}$ matrices are shown in $(14,15)$. From (14), the resistor admittances $g_{1}$ and $g_{2}$ and capacitor values $c_{1}$ and $c_{2}$ correspond to the equivalent damping and mass values $b_{1}^{\prime}, b_{2}^{\prime}, m_{1}{ }_{l}$ and $m_{2}{ }_{2}$ (16) respectively.

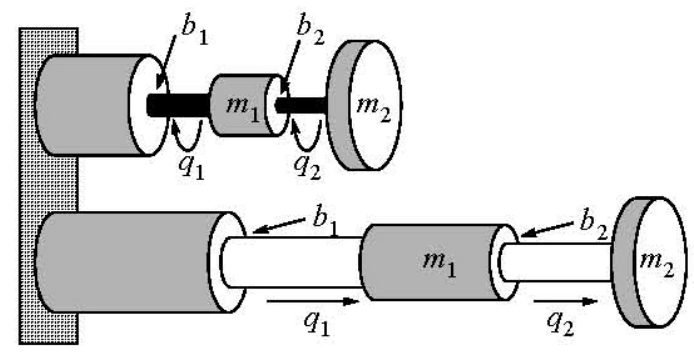

Figure 8: Redundant rotary \& prismatic actuators.

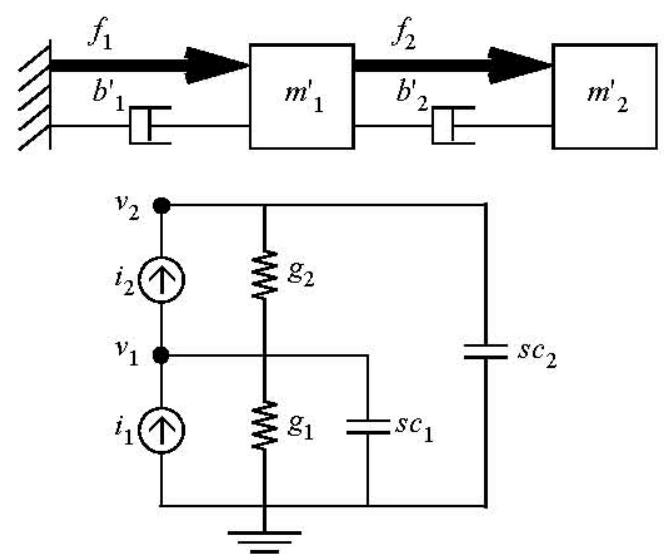

Figure 9: System models of redundant actuators.

$$
\begin{aligned}
& {\left[\begin{array}{c}
i_{1}-i_{2} \\
i_{2}
\end{array}\right]=\left[\begin{array}{cc}
g_{1}+g_{2} & -g_{2} \\
-g_{2} & g_{2}
\end{array}\right]\left[\begin{array}{l}
v_{1} \\
v_{2}
\end{array}\right]+\left[\begin{array}{cc}
c_{1} & 0 \\
0 & c_{2}
\end{array}\right] s\left[\begin{array}{l}
v_{1} \\
v_{2}
\end{array}\right]} \\
& {\left[\begin{array}{c}
f_{1}-f_{2} \\
f_{2}
\end{array}\right]=B^{\prime}\left[\begin{array}{c}
r_{1} \\
r_{1}+r_{2}
\end{array}\right]+M s\left[\begin{array}{c}
r_{1} \\
r_{1}+r_{2}
\end{array}\right]} \\
& B^{\prime}=\left[\begin{array}{cc}
b_{1}^{\prime}+b_{2}^{\prime} & -b_{2}^{\prime} \\
-b_{2}^{\prime} & b_{2}^{\prime}
\end{array}\right]=\left[\begin{array}{cc}
b_{1}+b_{2} & -b_{2} \\
-b_{2} & b_{2}
\end{array}\right] \\
& M=\left[\begin{array}{cc}
m_{1}^{\prime} & 0 \\
0 & m_{2}^{\prime}
\end{array}\right]=\left[\begin{array}{cc}
m_{1}+m_{2} & 0 \\
0 & m_{2}
\end{array}\right] \\
& {\left[\begin{array}{c}
b_{1}^{\prime} \\
b_{2}^{\prime} \\
m_{1}^{\prime} \\
m_{2}^{\prime}
\end{array}\right]=\left[\begin{array}{c}
b_{1} \\
b_{2} \\
m_{1}+m_{2} \\
m_{2}
\end{array}\right]}
\end{aligned}
$$


In this simple example, masses are sufficient to model the system behaviour but only because the device has a single degree of freedom so $M^{\prime}$ is diagonal and there is no cross-coupling between actuators. In general, however, effective mass is not always decoupled and the off-diagonal elements of $M^{\prime}$ can be expected to be non-zero. When $M^{\prime}$ is not diagonal, conventional single-terminal masses are unable to model the entire effective mass of the system. They can not model the off-diagonal terms that describe inertial effects resulting from relative motion of the actuators.

\subsection{Serial 2-DOF Robot}

Consider the 2-DOF serial robot shown in Figure 10. The mass matrix for this mechanism is approximated in (Craig, 1989) by two point masses $d_{1}$ and $d_{2}$ placed at the distal actuator and end-effector as indicated below. The resulting mass matrix (17) has the terms shown in (18-20) where $q_{1}$ and $q_{2}$ are the joint angles and $l_{1}$ and $l_{2}$ are the link lengths. Just as in the previous example, actuator damping coefficients $b_{1}$ and $b_{2}$ are taken to dominate the total system damping.

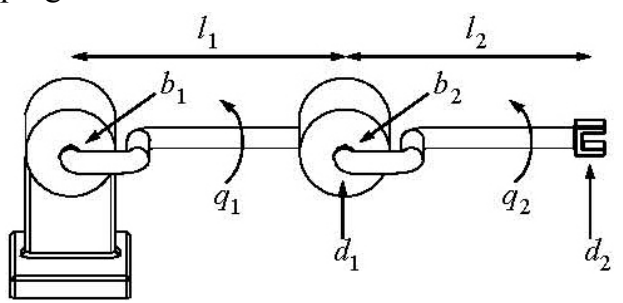

Figure 10: 2-DOF serial robot.

$$
\begin{gathered}
M(q)=\left[\begin{array}{ll}
m_{1}(q) & m_{3}(q) \\
m_{3}(q) & m_{2}(q)
\end{array}\right] \\
m_{1}=l_{2}^{2} d_{2}+2 l_{1} l_{2} d_{2} \cos \left(q_{2}\right)+l_{1}^{2}\left(d_{1}+d_{2}\right) \\
m_{2}=l_{2}^{2} d_{2} \\
m_{3}=l_{2}^{2} d_{2}+l_{1} l_{2} d_{2} \cos \left(q_{2}\right)
\end{gathered}
$$

The equivalent circuit model of this system is shown in Figure 11. It is similar to Figure 9 except that the capacitor values are configuration dependent and a third capacitor $c_{12}$ is included to model the coupled mass terms that are present. Performing nodal analysis results in (21) and the corresponding $M^{\prime}$ matrix in (22) which can be rearranged to solve for the mechanical model parameters in terms of the physical mass values in (23). $B^{\prime}$ is the same diagonal matrix as in (14).

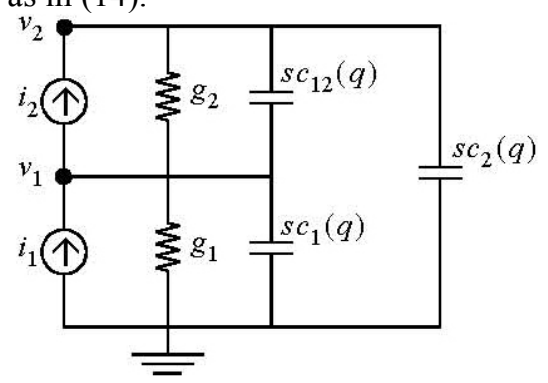

Figure 11: Electrical model of 2-DOF serial robot.

$$
\begin{gathered}
{\left[\begin{array}{c}
i_{1}-i_{2} \\
i_{2}
\end{array}\right]=\left[\begin{array}{cc}
g_{1}+g_{2} & -g_{2} \\
-g_{2} & g_{2}
\end{array}\right]\left[\begin{array}{l}
v_{1} \\
v_{2}
\end{array}\right]+\left[\begin{array}{cc}
c_{1}+c_{12} & -c_{12} \\
-c_{12} & c_{2}+c_{12}
\end{array}\right] s\left[\begin{array}{l}
v_{1} \\
v_{2}
\end{array}\right]} \\
M(q)=\left[\begin{array}{cc}
m_{1}^{\prime}+p_{12}^{\prime} & -p_{12}^{\prime} \\
-p_{12}^{\prime} & m_{2}^{\prime}+p_{12}^{\prime}
\end{array}\right]=\left[\begin{array}{ll}
m_{1}+m_{2} & m_{3}-m_{2} \\
m_{3}-m_{2} & m_{2}
\end{array}\right](22) \\
{\left[\begin{array}{c}
m_{1}^{\prime} \\
m_{2}^{\prime} \\
p_{12}^{\prime}
\end{array}\right]=\left[\begin{array}{c}
m_{1}+m_{3} \\
m_{3} \\
m_{2}-m_{3}
\end{array}\right]}
\end{gathered}
$$

Note from (22) that $M^{\prime}$ is diagonal (i.e. $p^{\prime}{ }_{12}=0$ ) when $p_{12}^{\prime}=0$. From $(19,20)$, this is merely the special case when $q_{2}= \pm \pi / 2$. Therefore, it is not possible to model this system using only masses due to their implicit ground reference, as described in Section 2.1. The off-diagonal terms can, however, be modelled using the MP model proposed in Section 2.2. It results in a mechanical system model that is topologically identical to the equivalent circuit in Figure 11 where each grounded capacitor $\left(c_{1}, c_{2}\right)$ is replaced by a regular mass and each ungrounded capacitor $\left(c_{12}\right)$ is replaced by an MP model since the MP model is able to accommodate a non-zero reference acceleration. The resulting mechanical system is shown in Figure 12.

Although $p^{\prime}{ }_{12}$ has a negative value when $-\pi / 2<q_{2}<\pi / 2$, the net mass perceived by each actuator is always positive because $M$ is positive definite. When $p_{12}^{\prime}$ is negative, it simply means that the motion of actuator 1 reduces the net mass perceived by actuator 2 , but the net mass perceived by actuator 2 is always greater than zero.

\subsection{Parallel 2-DOF Robot}

The same technique can be applied to parallel manipulators such as the 2-DOF 5-bar linkage used 
by (Hayward et al., 1994). In the case of parallel manipulators, each actuator is referenced to ground but there remains a coupling between the effective mass perceived by each actuator which, like a serial manipulator, is configuration dependent. This coupling is modelled by $c_{12}$ and $p_{12}^{\prime}$ in the equivalent electrical and mechanical models shown in Figure 13. Typically, parallel manipulators also have coupled damping terms due to their passive joints which would be modelled by a conductance $g_{12}$ added between nodes 1 and 2 (i.e. in parallel with $c_{12}$ ). However, for the sake of simplicity, the damping of the passive joints are neglected here.

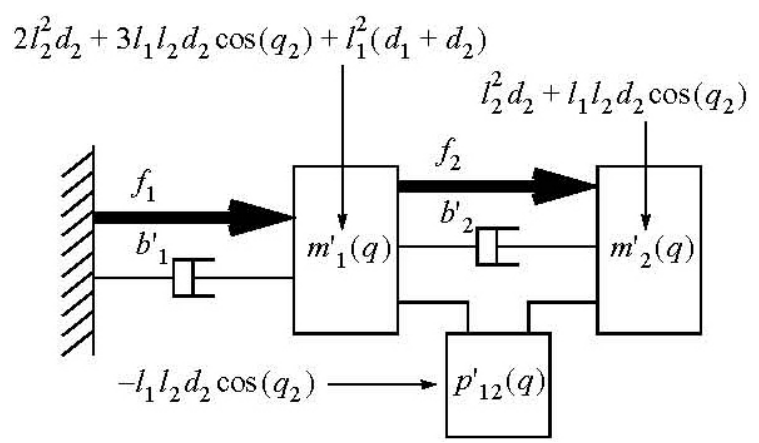

Figure 12: Mechanical model of a 2-DOF serial robot.
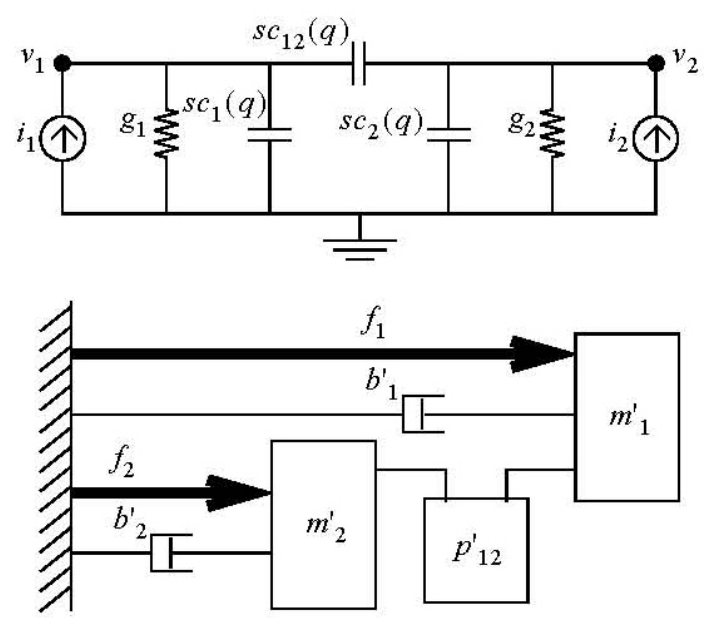

Figure 13: Model of a 2-DOF parallel robot.

Performing nodal analysis on the circuit in Figure 13 results in (24) by inspection. For a parallel robot, currents and voltages correspond directly to joint forces and joint rates so $B^{\prime}=B$ and $M^{\prime}=M$. For a mass matrix of the form shown in (17), the elements of the $M$ matrix, and therefore the parameter values associated with the masses and MP models of Figure 13 , are shown in (26).

$$
\begin{gathered}
{\left[\begin{array}{l}
i_{1} \\
i_{2}
\end{array}\right]=\left[\begin{array}{ll}
g_{1} & 0 \\
0 & g_{2}
\end{array}\right]\left[\begin{array}{l}
v_{1} \\
v_{2}
\end{array}\right]+\left[\begin{array}{cc}
c_{1}+c_{12} & -c_{12} \\
-c_{12} & c_{2}+c_{12}
\end{array}\right] s\left[\begin{array}{l}
v_{1} \\
v_{2}
\end{array}\right]} \\
{\left[\begin{array}{l}
f_{1} \\
f_{2}
\end{array}\right]=\left[\begin{array}{cc}
b_{1}^{\prime} & 0 \\
0 & b_{2}^{\prime}
\end{array}\right]\left[\begin{array}{l}
r_{1} \\
r_{2}
\end{array}\right]+\left[\begin{array}{cc}
m_{1}^{\prime}+p_{12}^{\prime} & -p_{12}^{\prime} \\
-p_{12}^{\prime} & m_{2}^{\prime}+p_{12}^{\prime}
\end{array}\right] s\left[\begin{array}{l}
r_{1} \\
r_{2}
\end{array}\right]} \\
{\left[\begin{array}{l}
m_{1}^{\prime} \\
m_{2}^{\prime} \\
p_{12}^{\prime}
\end{array}\right]=\left[\begin{array}{c}
m_{1}+m_{3} \\
m_{2}+m_{3} \\
-m_{3}
\end{array}\right]}
\end{gathered}
$$

\subsection{Multiple DOF Robots}

This technique is easily extended to devices with any number $n$ of degrees of freedom. With serial manipulators, the compliance and damping is often mainly in the actuators and the damping $B$ and spring $K$ matrices are diagonal $(27,28)$. With parallel manipulators, the $B$ and $K$ matrices typically contain off-diagonal terms but they are easily modelled using conventional techniques since springs and dampers are 2-terminal devices which can be placed at any two nodes in a system diagram.

$$
\begin{gathered}
B=\operatorname{diag}\left(\left[\begin{array}{llll}
b_{1} & b_{2} & \ldots & b_{n}
\end{array}\right]\right) \\
K=\operatorname{diag}\left(\left[\begin{array}{lllll}
1 / k_{1} & 1 / k_{2} & \ldots & 1 / k_{n}
\end{array}\right]\right)
\end{gathered}
$$

To account for inertial cross-coupling, the model must contain a capacitor and/or MP model between every pair of actuators. For example, the electric circuit model and corresponding mechanical system model of a serial 3-DOF manipulator are shown in Figure 14. The capacitance $C$ matrix resulting from the nodal analysis (29) of the circuit in Figure 14 is shown in (30).

$$
\left[\begin{array}{c}
i_{1}-i_{2} \\
i_{2}-i_{3} \\
i_{3}
\end{array}\right]=G(q)\left[\begin{array}{c}
v_{1} \\
v_{2} \\
v_{3}
\end{array}\right]+C(q) s\left[\begin{array}{c}
v_{1} \\
v_{2} \\
v_{3}
\end{array}\right]
$$

$C(q)=\left[\begin{array}{ccc}c_{1}+c_{12}+c_{13} & -c_{12} & -c_{13} \\ -c_{12} & c_{2}+c_{12}+c_{23} & -c_{23} \\ -c_{13} & -c_{23} & c_{3}+c_{23}+c_{13}\end{array}\right]$

Just as in the previous examples, the $3 \times 3$ mass matrix $M^{\prime}$ (32) is rearranged into the form shown in (31) to parallel the current/voltage relationship of (29). For the mass matrix $M$ of the form shown in (33), the entries of the $M^{\prime}$ matrix are solved for in (34). 

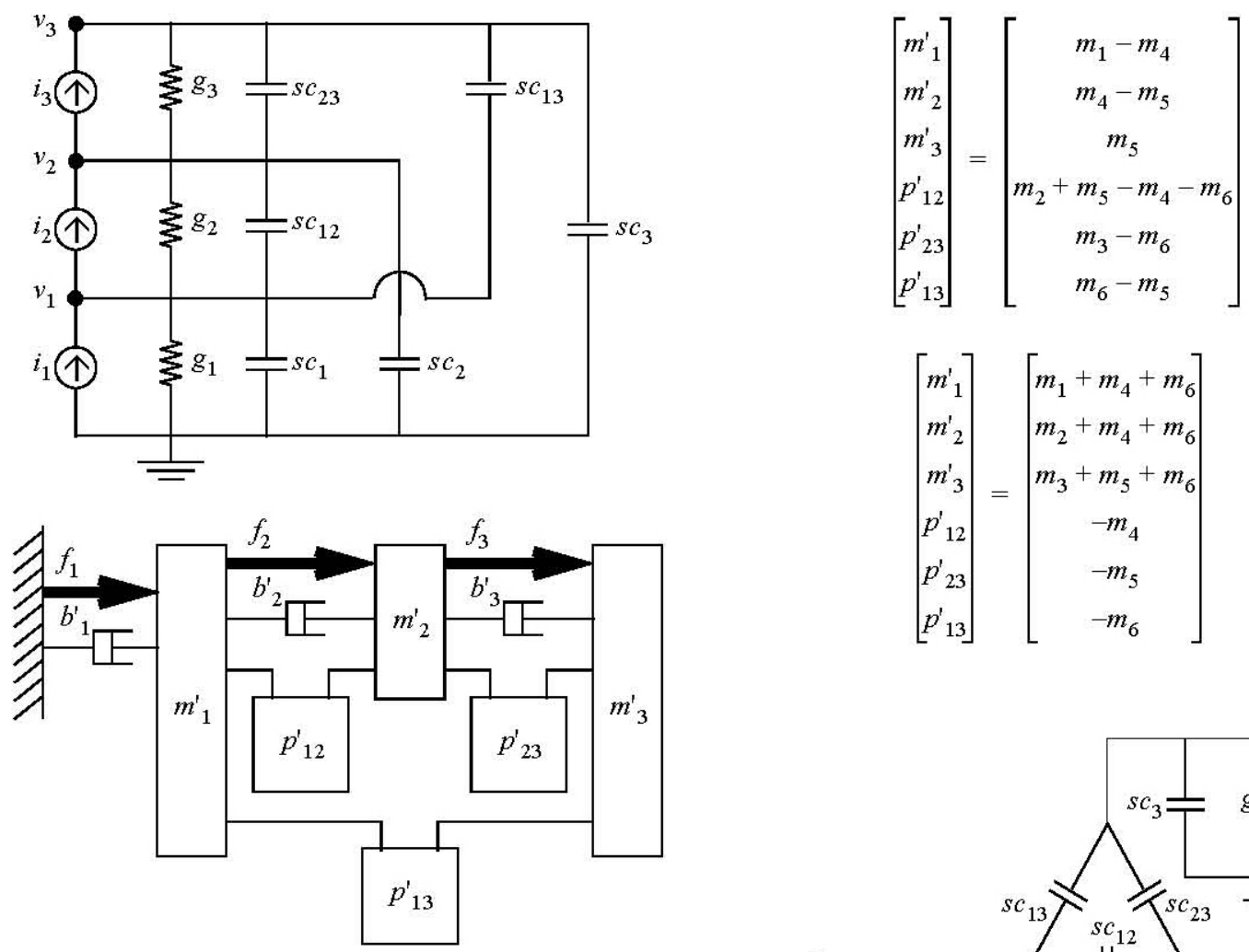

$\left[\begin{array}{c}m_{1}^{\prime} \\ m_{2}^{\prime} \\ m_{3}^{\prime} \\ p_{12}^{\prime} \\ p_{23}^{\prime} \\ p_{13}^{\prime}\end{array}\right]=\left[\begin{array}{c}m_{1}+m_{4}+m_{6} \\ m_{2}+m_{4}+m_{6} \\ m_{3}+m_{5}+m_{6} \\ -m_{4} \\ -m_{5} \\ -m_{6}\end{array}\right]$

Figure 14: Model of a 3-DOF serial robot.

Similarly, for a parallel 3-DOF robot, the electric circuit model and corresponding mechanical system model are shown in Figure 15. For a mass matrix of the form shown in (33), the elements of $M$ are shown in (35).

$$
\left[\begin{array}{c}
f_{1}-f_{2} \\
f_{2}-f_{3} \\
f_{3}
\end{array}\right]=B^{\prime}\left[\begin{array}{c}
r_{1} \\
r_{1}+r_{2} \\
r_{1}+r_{2}+r_{3}
\end{array}\right]+M s\left[\begin{array}{c}
r_{1} \\
r_{1}+r_{2} \\
r_{1}+r_{2}+r_{3}
\end{array}\right]
$$

$M(q)=$

$\left[\begin{array}{ccc}m_{1}^{\prime}+p_{12}^{\prime}+p_{13}^{\prime} & -p_{12}^{\prime} & -p_{13}^{\prime} \\ -p_{12}^{\prime} & m_{2}^{\prime}+p_{12}^{\prime}+p_{23}^{\prime} & -p_{23}^{\prime} \\ -p_{13}^{\prime} & -p_{23}^{\prime} & m_{3}^{\prime}+p_{13}^{\prime}+p_{23}^{\prime}\end{array}\right]$

$$
M(q)=\left[\begin{array}{lll}
m_{1}(q) & m_{4}(q) & m_{5}(q) \\
m_{4}(q) & m_{2}(q) & m_{6}(q) \\
m_{5}(q) & m_{6}(q) & m_{3}(q)
\end{array}\right]
$$

\section{CONCLUSION}

It is argued that a plain mass is not a complete and general model of a capacitor since a mass only has 
one terminal whereas a capacitor has two. The response of a mass corresponds to its acceleration with respect to ground and, therefore, can only be used to simulate a capacitor which has one terminal connected to ground. It cannot be used to simulate a capacitor which has a non-zero reference voltage. A new model described here that consists of a mass and a pulley correctly simulates the response of a capacitor in the general case.

It is shown that the MP model can be used to model systems with cross-coupled effective masses which are otherwise, impossible to model with pure masses alone. This includes both serial and parallel manipulators with any number of degrees of freedom. The mechanical system model that is obtained fully describes the dynamic response of the system and is topologically identical to its electric circuit equivalent. As shown in (Stocco \& Yedlin, 2006), this makes it possible to apply electric circuit analysis techniques to mechanical systems, directly.

\section{ACKNOWLEDGEMENTS}

The authors gratefully acknowledge Tim Salcudean for his valuable comments during the preparation of this manuscript.

\section{REFERENCES}

Brune, O., 1931. "Synthesis of a finite two-terminal network whose driving-point impedance is a prescribed function of frequency". J. Math. Physics. vol. 10, pp. 191-236.

Craig, J.J., 2005. "Introduction to Robotics Mechanics and Control". 3rd ed., Pearson Prentice Hall.

Eppinger, S., Seering, W., 1992. "Three Dynamic Problems in Robot Force Control". IEEE Trans. Robotics \& Auto., V. 8, No. 6, pp. 751-758.

Fairlie-Clarke, A.C., 1999. "Force as a Flow Variable". Proc. Instn. Mech. Engrs., V. 213, Part I, pp. 77-81.

Foster, R. M., 1924. "A reactance theorem". Bell System Tech. J., vol. 3, pp. 259-267.

Hamill, D.C., 1993. "Lumped Equivalent Circuits of Magnetic Components: The Gyrator-Capacitor Approach". IEEE Transactions on Power Electronics, vol. 8, pp. 97.

Hayward, V., Choksi, J., Lanvin, G., Ramstein, C., 1994. "Design and Multi-Objective Optimization of a Linkage for a Haptic Interface”. Proc. of ARK '94, 4th Int. Workshop on Advances in Robot Kinematics (Ljubliana, Slovenia), pp. 352-359.

Paynter, H.M., 1961. Analysis and Design of Engineering Systems. MIT Press.
Sass, L., McPhee, J., Schmitke, C., Fisette, P., Grenier, D., 2004. "A Comparison of Different Methods for Modelling Electromechanical Multibody Systems". Multibody System Dynamics, vol. 12, pp. 209-250.

Stocco, L., Yedlin, M., Sept. 2006. "Closing the Loop on the Electro-Mechanical System Analogy". Submitted to: IEEE J. Circuits \& Systems.

Tilmans, H.A.C., 1996. "Equivalent circuit representation of electromechanical transducers: I. Lumpedparameter systems". J. Micromech. Microeng, vol. 6, pp. 157-176.

van Amerongen, J., Breedveld, P., 2003. "Modelling of physical systems for the design and control of mechatronic systems". Annual Reviews in Control, vol. 27, pp. 87-117.

Yamakita, M., Shibasato, H., Furuta, K., 1992. "TeleVirtual Reality of Dynamic Mechanical Model". Proc. IEEE/RSJ Int. Conf. Intelligent Robots \& Systems, (Raleigh, NC), pp. 1103,-1110. 